\title{
Franck-Condon Factors and r-Centroids For Several Systems of CoF Molecule
}

\author{
Nadhem Q. M. ${ }^{1, a^{*}}$, Supriya Behere ${ }^{2, b}$ and Behere S. H. ${ }^{3, c}$ \\ 1,2,3 Deparment of Physics, Dr. B. A. M. University, Aurangabad 431004 , India \\ a nadhem.physics@gmail.com, ${ }^{\mathrm{b}}$ supriyabehere1987@gmail.com, ${ }^{\mathrm{c}}$ subhashbehere@gmail.com
}

\begin{abstract}
Keywords: Transition Probability Parameters; Franck-Condon Factors; r-centroids; CoF; Transition Metals.
\end{abstract}

ABSTRACT. Franck-Condon factors factors and r-centroids have been calculated for the $G^{3} \Phi_{4}-$ $\mathrm{X}^{3} \Phi_{4}, \mathrm{~K}^{3} \Phi_{4}-\mathrm{X}^{3} \Phi_{4}, \mathrm{~L}^{3} \Phi_{4}-\mathrm{X}^{3} \Phi_{4},[20.6]^{3} \Gamma_{5}-\mathrm{X}^{3} \Phi_{4}$ and $[34.7]^{3} \Gamma_{5}-\mathrm{X}^{3} \Phi_{4}$ bands of Cobalt monofluoride ${ }^{59} \mathrm{Co}^{19} \mathrm{~F}$ from the best available molecular constants using Morse and RydbergKlein-Rees (RKR) methods. The reliability of the obtained values was verified by comparing the results of five different programs. Based on the calculated Franck-Condon factors values, the appearance of the bands in these systems are discussed and the relationship between the r-centroids and the band-origin wavenumbers and wavelengths are also determined along with accuracy factors.

\section{INTRODUCTION}

Franck-Condon factors (FCFs) are important parameters for every molecular band system, since they are involved in the calculation of the relative band intensities, the determination of the molecular structure, population of the levels in the upper electronic state involved in transition and the calculation of radiative lifetimes, vibrational temperature, kinetics of energy transfer, etc [1]. The FCFs are also useful in the studies of radiative transfer in the atmospheres of stellar and other astronomical objects, which contain molecular species [2]. On the other hand, the $r$-centroid [3-5] also has been found of much importance as it is used in the interpretation of intensity measurements of many band systems of astrophysically important molecules and, to determine the variation of the electronic transition moment with inter-nuclear separation, and thereby to derive band strengths, and other molecular transition probability parameters [6].

"The properties of transition metal compounds have fascinated physicists and chemists for a long time. These compounds have an enormous potential for future applications of solar energy conversion, information storage systems, chemical or biochemical sensors, low dimensional semiconductors, supramolecular systems, chemical synthesis, etc"[7]. In astrophysics it is the transition-metal-containing compounds are very important [8-10]. More over the presence of transition metal elements in cool stars has been known for some time [11].

In recent years, enough progress has been made in an understanding of the electronic structure of transition metal oxides and hydrides. Metal fluorides are also potential stellar and interstellar molecules, although Florin has relatively small cosmic abundance. Metal fluorides also have strong bonds, which favor their formation in stellar atmospheres [12]. In this paper, the FCFs and $r$-centroids for the bands of the $\mathrm{G}^{3} \Phi_{4}-\mathrm{X}^{3} \Phi_{4}, \mathrm{~K}^{3} \Phi_{4}-\mathrm{X}^{3} \Phi_{4}, \mathrm{~L}^{3} \Phi_{4}-\mathrm{X}^{3} \Phi_{4},[20.6]^{3} \Gamma_{5}-$ $\mathrm{X}^{3} \Phi_{4}$ and $[34.7]^{3} \Gamma_{5}-\mathrm{X}^{3} \Phi_{4}$ systems of the ${ }^{59} \mathrm{Co}^{19} \mathrm{~F}$ molecule are reported. To the best of our knowledge, such information is not available in the literature. Therefore the reliable values of FCFs and $r$-centroids for these band systems have been determined using more accurate numerical integration procedure and suitable potentials. The required molecular constants are taken from the works of [8-12]. 


\section{FRANCK CONDON FACTORS AND R-CENTROID}

Within the framework of the Born-Oppenheimer approximation, the expression for band intensities in emission is given in as [13]:

$$
I_{e m}\left(v^{\prime} v^{\prime \prime}\right)=\frac{64}{3} \pi^{4} C N_{v^{\prime}} v^{4}{\overline{R_{e}(r)}}^{2}\left[\int \psi_{v^{\prime}}(r) \psi_{v^{\prime \prime}}(r) d r\right]^{2}
$$

Where $\mathrm{C}$ is velocity of light, $N_{v^{\prime}}$ is number of molecules in $v^{\prime}$ th level, $\boldsymbol{v}$ is the wave number of radiation of the $v^{\prime} \rightarrow v^{\prime \prime}$ vibronic transition; and $\overline{R_{e}(r)}$ is the average transition moment, which is commonly represented as a function of internuclear distance $r . \psi_{v^{\prime}}(r)$ and $\psi_{v^{\prime \prime}}(r)$ are the wave functions which correspond to the rotationless $(J=0)$ vibrational levels in the upper and lower electronic states, respectively. They satisfy the time-independent Schrödinger radial wave equation:

$$
\left[-\frac{\hbar^{2}}{2 \mu} \frac{d^{2}}{d r^{2}}+V(r)\right] \psi_{v}(r)=E_{v} \psi_{v}(r)
$$

In which $r$ is the inter-nuclear distance, $\hbar$ is Planck's constant, $\mu$ is the reduced mass of the two atoms forming the molecule and $V(r)$ is the potential energy.

For many band systems, $R_{e}(r)$ can be well approximated by a constant or a linear function of $r$. Since the wave-mechanical formulation of the Franck-Condon principle rests on the assumption that this variation is slow. In such a situation, the $r$-centroid approximation can be employed. The quantity in the square bracket appearing in eq. (1) is the square of overlap integral known as Franck-Condon factor which is usually denoted by $q_{v^{\prime}} v^{\prime \prime}$ and determines how the intensity is distributed among the vibrational bands of a band system. It is expressed as,

$$
q_{v^{\prime} v^{\prime \prime}}=\left[\int \psi_{v^{\prime}}(r) \psi_{v^{\prime \prime}}(r) d r\right]^{2}
$$

$r$-Centroid is the $r$-coordinate of the centroid of the area represented by the overlap integral and defined as:

$$
\bar{r}_{v^{\prime} v^{\prime \prime}}=\frac{\int \psi_{v^{\prime}}(r) r \psi_{v^{\prime \prime}}(r) d r}{\int \psi_{v^{\prime}}(r) \psi_{v^{\prime \prime}}(r) d r}
$$

In the above equation $\bar{r}$ is the $r$-centroid which is the weighted average with respect to $\psi_{v^{\prime}} \psi_{v^{\prime \prime}}$ of the range of internuclear separation $r$ values experienced by the molecule in both states of the $v^{\prime} \rightarrow v^{\prime \prime}$ transition [11] or may be looked upon as an effective value of $r$ for the band in much the same way as the $v$ of eqn.(1) constitutes its effective wave number [14]. The r-centroid is the most practical method for calculation of the band strengths of the observed transitions in diatomic molecules .Since it is an approximation, there are limits to its use. According to [9] the approximation is good to the $n$th order if:

$$
\bar{r}_{v^{\prime} v^{\prime \prime}}=\int \psi_{v^{\prime}}(r) r^{n} \psi_{v^{\prime \prime}}(r) d r / \int \psi_{v^{\prime}}(r) r^{n-1} \psi_{v^{\prime \prime}}(r) d r
$$

and its accuracy factor that indicates the degree of its applicability given by Drake [15].

$$
Y_{v^{\prime} v^{\prime \prime}}^{(n)}=\bar{r}_{v^{\prime} v^{\prime \prime}} /\left(\bar{r}_{v^{\prime} v^{\prime \prime}}\right)^{n} \int \psi_{v^{\prime}}(r) r^{n} \psi_{v^{\prime \prime}}(r) d r /\left(\bar{r}_{v^{\prime} v^{\prime \prime}}\right)^{n} \int \psi_{v^{\prime}}(r) \psi_{v^{\prime \prime}}(r) d r \cong 1
$$

When $Y_{v^{\prime} v^{\prime \prime}}^{(n)}$ is unity the approximation implied in equations (4) and (6) is exact. Deviation from unity for $(n \geq 2)$ is a measure of the error in the approximation.

The accuracy of the calculated FCFs and $r$-centroids depend on knowledge of the potential energy function of the lower and upper electronic states, which are used to solve the radial Schrödinger 
equation for rotationless vibrational wavefunctions $\psi_{v^{\prime}}(r)$ and $\psi_{v^{\prime \prime}}(r)$. The commonly used potentials are Morse [16] and Rydberg-Klein-Rees (RKR) [17-19].

\section{COMPUTATIONAL METHOD}

In the present work we use the same computational methods as described in our previous work [20]. where the results are given for each method by mentioning the name of the author in the following order: (1) Le Roy[21-22], (2) Telle[23], (3) Espy[24], (4) Jarmain and McCallum[25] referred as Jarmain and (5) Felenbok[26].

\section{RESULTS AND DISCUSSION}

Using above mentioned approaches, FCFs and $r$-centroids arrays have been computed for the following Systems: G-X, K-X, L-X, [20.6] ${ }^{3} \Gamma_{5}-\mathrm{X}$ and $[34.7]^{3} \Gamma_{5}-\mathrm{X}$ of cobalt monofluoride. Tables 2, 3,4,5 and 6, show the results obtained using the approach based on Espy RKR/FCF computer programmes along with the wave number, wavelengths and accuracy factor of $r$ centroides for $n=3$. The Comparison of the FCFs and $r$-centroides calculated using the five approaches for G-X system is presented in Table 7 and 8 . In these tables the values are calculated for many bands which were not experimentally observed.

The $\Delta \boldsymbol{r}_{\boldsymbol{e}}=\boldsymbol{r}_{\boldsymbol{e}}^{\prime}-\boldsymbol{r}_{\boldsymbol{e}}^{\prime \prime}$ for the band systems G-X, K-X, L-X, [20.6] ${ }^{3} \Gamma_{5}-\mathrm{X}$ and $[34.7]^{3} \Gamma_{5}-\mathrm{X}$ are: $0.088,0.027,0.026,0.045$ and 0.016 respectively. These small values indicate that the minima of the potential energy curves involved in K-X, L-X and $[34.7]^{3} \Gamma_{5}-X$ transitions lie one above the other (Fig.1) and their Condon parabola is very narrow, appear to lie along the main diagonal $\boldsymbol{v}^{\prime}=\boldsymbol{v}^{\prime \prime}$ in the Deslandres table and has its maximum value at the $(0,0)$ band. In a three dimensional representation, the FCFs surface looks like a diagonal ridge along $\boldsymbol{v}^{\prime}=\boldsymbol{v}^{\prime \prime}$. The situation is somewhat different for $[20.6]^{3} \Gamma_{5}-\mathrm{X}$ band system in which $\Delta \boldsymbol{r}_{\boldsymbol{e}}$ is a little larger $(\sim 0.05 \AA)$, the ridge widens into a very narrow parabolic ridge with an axial valley. For G-X band system $\Delta \boldsymbol{r}_{\boldsymbol{e}}$ is relatively larger and the Condon parabola widens and its vertex moves right the prime diagonal away from $(0,0)$ in accordance with the observations of Nicholls [27]. Condon parabola is a locus of intense bands in $\boldsymbol{v}^{\prime \prime}$ progressions which often pass through $(0,0)$ and are not completely symmetric about $\boldsymbol{v}^{\prime}=\boldsymbol{v}^{\prime \prime}$ unless the $\boldsymbol{\omega}_{\mathbf{e}}^{\prime}$ and $\boldsymbol{\omega}_{\mathbf{e}}^{\prime \prime}$ are very close together [28]. FCFs of such bands are shown in bold figures in corresponding tables.

The FCFs of G-X show that the $(0,1),(0,0)$ and $(1,0)$ bands are the strongest of all the observed bands. Some bands are found to be roughly equal in magnitude such as $[(0,2),(2,0),(3,1)]$ and $[(2,1),(4,1),(5,2)]$ bands which indicate that these bands of comparable intensities. FCFs for the bands $(1,2),(3,3),(3,5),(4,4),(4,6),(6,2)$ and $(6,3)$ are likely to be intense, hence one expects that these bands may be observed experimentally where as other bands, having small FCFs may not be observed experimentally. Refer to Fig.2. The FCFs of K-X, L-X, $[20.6]^{3} \Gamma_{5}-X$ and $[34.7]^{3} \Gamma_{5}-X$ systems show that the $(0,0)$ is the strongest of all the observed bands. The $\Delta \boldsymbol{v}=0, \pm 1$ bands are intense compared to others, suggesting their presence in the observed spectrum.

The FCFs generated using Morse potential show deviations with those generated by RKR potentials for most of the transitions even for low vibrational levels and these differences for most intense observed bands in G-X, K-X, L-X, [20.6 $]^{3} \Gamma_{\mathbf{5}}-\mathrm{X}$ and $[34.7]^{3} \Gamma_{\mathbf{5}}-\mathrm{X}$ are as follows : $1.74 \times \mathbf{1 0}^{-2}$, $1.29 \times \mathbf{1 0}^{-2}, 6.97 \times \mathbf{1 0}^{-3}, 2.07 \times \mathbf{1 0}^{-2}$ and $6.16 \times \mathbf{1 0}^{-3}$ respectively (approximately $0.01 \AA$ ). In case of r-centroids, these differences are: $2.66 \times \mathbf{1 0}^{-3}, 8.00 \times \mathbf{1 0}^{-\mathbf{4}}, 1.16 \times \mathbf{1 0}^{-\mathbf{3}}, 6.80 \times \mathbf{1 0}^{-\mathbf{4}}$ and $9.20 \times \mathbf{1 0}^{-4}$ respectively or approximately $0.001(\AA)$. These differences increase for higher levels in a $\mathbf{v}^{\prime \prime}$ or $\mathbf{v}^{\prime}$ progression. The FCFs differences may be explained in terms of deviation of theoretical value of rotational constant $\alpha$, computed using Pekeris relation [29] from corresponding experimental value. 
The percentage error (deviation) between the experimental and computed values of the rotational constant $\alpha$ for X, G, K, L, [20.6] ${ }^{3} \Gamma_{\mathbf{5}}$ and $[34.7]^{3} \Gamma_{\mathbf{5}}$ systems are: $46 \%, 15 \%, 4 \%, 19 \%, 15 \%$ and $8 \%$ respectively. This suggests that the lower part of the potential energy curves of the excited states may be described by a simple Morse function but it cannot describe the ground state properly. The r-centroid of the observed bands vary systematically from band to band of a system. In the case of very small overlap integrals, r-centroids have irregular behavior, and such values are not used in our consideration. The sequence difference $\Delta \overline{\boldsymbol{r}}=\boldsymbol{r}_{\boldsymbol{v}^{\prime}+\mathbf{1}, \boldsymbol{v}^{\prime \prime}+\mathbf{1}}-\boldsymbol{r}_{\boldsymbol{v}^{\prime} \boldsymbol{v}^{\prime \prime}}$ for the observed bands are found generally to be constant for the strong sequences. In G-X, L-X, $[\mathbf{2 0 . 6}]^{3} \Gamma_{\mathbf{5}}-\mathrm{X}$ and $[\mathbf{3 4 . 7}]^{\mathbf{3}} \Gamma_{\mathbf{5}}$ -X systems the sequence difference is found to be constant amounting to about $0.01(\AA)$. But in the case of K-X system, the sequence difference is not a constant and is varying from 0.004-0.01 ( $\AA$ ).

For all these band systems, it is found that the r-centroids increase systematically with increasing wavelength of the corresponding $\boldsymbol{v}^{\prime}, \boldsymbol{v}^{\prime \prime}$ bands, which is expected for transitions between states with $\boldsymbol{r}_{\boldsymbol{e}}^{\prime}>\boldsymbol{r}_{\boldsymbol{e}}^{\prime \prime}$ and the bands are red degraded. For example the wavelength and wavenumbers versus $\mathrm{r}$ centroids of few bands of $\Delta \boldsymbol{v}=3$ sequence of the G-X system are displayed in Table 9 and wavelength versus r-centroids are plotted in Fig. 3 which show a systematic relationship. Nicholls et al. [11] have shown that $\boldsymbol{r}_{\mathbf{0 , 0}}$ is usually greater than $\left(\boldsymbol{r}_{\boldsymbol{e}}^{\prime}+\boldsymbol{r}_{\boldsymbol{e}}^{\prime \prime}\right) / \mathbf{2}$ for systems whose potentials are not very anharmonic. In the present case, $\boldsymbol{r}_{\mathbf{0}, \mathbf{0}}$ is found to be slightly greater than $\left(\boldsymbol{r}_{\boldsymbol{e}}^{\prime}+\boldsymbol{r}_{\boldsymbol{e}}^{\prime \prime}\right) / \mathbf{2}$ by $0.004,0.005,0.005,0.004$ and 0.005 respectively, suggesting that the potentials are almost harmonic. The vibrational sum rule is satisfied for lower and upper states progressions up to $\left(2, \boldsymbol{v}^{\prime \prime}\right)$, $\left(\mathbf{v}^{\prime}, 0\right)$ progressions for G-X, $[\mathbf{2 0 . 6}]^{3} \Gamma_{\mathbf{5}}-\mathrm{X}$ and up to $\left(2, \mathbf{v}^{\prime \prime}\right),\left(\mathbf{v}^{\prime}, 1\right)$ progressions for $\mathrm{K}-\mathrm{X}, \mathrm{L}-\mathrm{X}$ and to $\left(2, \boldsymbol{v}^{\prime \prime}\right),\left(\boldsymbol{v}^{\prime}, 2\right)$ progressions for $[34.7]^{3} \Gamma_{\mathbf{5}}-\mathrm{X}$, indicating that further experimental work will not be helpful to observe more bands in these progressions. To assess the reliability of r-centroids values we have calculated the factor $\boldsymbol{Y}_{\boldsymbol{v}^{\prime} \boldsymbol{v}^{\prime \prime}}^{(\boldsymbol{n})}$ for $\mathrm{n}=3$. In terms of this factor $1 \%$ agreement means that this quantity, which should be exactly 1, is smaller than 1.01 or $\left|\mathbf{1}-\boldsymbol{Y}_{\boldsymbol{v}^{\prime} \boldsymbol{v}^{\prime \prime}}^{(\boldsymbol{n})}\right|<0.01$. According to McCallum [30] the obtained values may be considered reliable for which $\left|\mathbf{1}-\boldsymbol{Y}_{\boldsymbol{v}^{\prime} \boldsymbol{v}^{\prime \prime}}^{(3)}\right|<0.02$.

\section{Figures and Tables}

Table 1: Spectroscopic constants of the different states of ${ }^{59} \mathrm{Co}^{19} \mathrm{~F}$

\begin{tabular}{cllllll}
\hline Constant $^{\mathrm{a}}$ & \multicolumn{1}{c}{$X^{3} \Phi_{4}$} & \multicolumn{1}{c}{$G^{3} \Phi_{4}$} & \multicolumn{1}{c}{$K^{3} \Phi_{4}$} & \multicolumn{1}{c}{$L^{3} \Phi_{4}$} & {$[20.6]^{3} \Gamma_{5}$} & \multicolumn{1}{c}{$[34.7]^{3} \Gamma_{5}$} \\
\hline$T_{e}$ & 0 & 10385.4033 & 18912.2894 & 19252.1574 & 20679.3781 & 34694.0474 \\
$T_{0}$ & $-338.8650^{b}$ & $10046.5383^{c}$ & $18573.4244^{d}$ & $18913.2924^{e}$ & $20340.5131^{f}$ & $34355.1824^{g}$ \\
$\omega_{e}$ & 677.59 & 580.3556 & 673.72 & 646.37 & 630.54 & 685.65 \\
$\omega_{e} x_{e}$ & 2.16 & 2.72447 & 4.28 & 2.76 & 3.40 & 3.61 \\
$\omega_{e} y_{e}$ & -0.161 & 0.007257 & - & - & - & - \\
$B_{e}$ & 0.389824 & 0.3530152 & 0.3781 & 0.3785 & 0.3702 & 0.3829 \\
$\alpha_{e}$ & 0.003317 & 0.0026928 & 0.0029 & 0.0028 & 0.0023 & 0.0029 \\
$\alpha_{e}$ Calc. ${ }^{h}$ & 0.001822 & 0.002291 & 0.003010 & 0.002261 & 0.002648 & 0.002656 \\
$r_{e}(\AA)$ & 1.734924 & 1.823137 & 1.761624 & 1.760690 & 1.780322 & 1.750546 \\
Ref & {$[12]$} & {$[9]$} & {$[10-11]$} & {$[10-11]$} & {$[10]$} & {$[8]$} \\
\hline
\end{tabular}

a All values are in data units of $\mathrm{cm}^{-1}$, unless otherwise noted .

$b$ Adjusted $T_{e}$ of the $\mathrm{X}$ state to give $T_{0}=0 \mathrm{~cm}^{-1}$.

$c, d, e, f, g$ Adjusted $T_{e}$ of the state to give the corresponding $v_{00}$ value of [9],[10],[11] and [8]. $h \alpha_{e}$ calculated using the Pekeris relation. 
Table 2: Transition Probability parameters For $G^{3} \Phi_{4}-X^{3} \Phi_{4}$ band system of ${ }^{59} \mathrm{Co}^{19} \mathrm{~F}$

\begin{tabular}{|c|c|c|c|c|c|c|c|c|}
\hline $\boldsymbol{v}^{\prime} \backslash \boldsymbol{v}^{\prime \prime}$ & $\mathbf{0}$ & 1 & 2 & 3 & 4 & 5 & 6 & $\Sigma_{\boldsymbol{v}^{\prime \prime}} \boldsymbol{q}_{v^{\prime} v^{\prime \prime}}$ \\
\hline \multirow[t]{5}{*}{$\mathbf{0}$} & 10336.670 & 9663.919 & 8996.942 & 8336.699 & 7684.157 & 7040.282 & 6406.041 & \\
\hline & 0.967 & 1.035 & 1.112 & 1.200 & 1.301 & 1.420 & 1.561 & \\
\hline & 0.357 & 0.400 & 0.188 & 0.048 & 0.007 & 0.001 & 0.000 & 1.000 \\
\hline & 1.783 & 1.829 & 1.879 & 1.935 & 1.998 & 2.072 & 2.174 & \\
\hline & 0.002 & 0.000 & 0.002 & 0.004 & 0.006 & 0.010 & 0.016 & \\
\hline \multirow[t]{5}{*}{1} & 10911.600 & 10238.850 & 9571.871 & 8911.629 & 8259.087 & 7615.212 & 6980.970 & \\
\hline & 0.917 & 0.977 & 1.045 & 1.122 & 1.211 & 1.313 & 1.433 & \\
\hline & 0.339 & 0.000 & 0.225 & 0.285 & 0.122 & 0.026 & 0.003 & 1.000 \\
\hline & 1.743 & 1.640 & 1.840 & 1.889 & 1.944 & 2.008 & 2.084 & \\
\hline & 0.000 & 0.213 & 0.003 & 0.000 & 0.003 & 0.006 & 0.009 & \\
\hline \multirow[t]{5}{*}{2} & 11481.140 & 10808.400 & 10141.420 & 9481.176 & 8828.634 & 8184.759 & 7550.517 & \\
\hline & 0.871 & 0.925 & 0.986 & 1.055 & 1.133 & 1.222 & 1.324 & \\
\hline & 0.186 & 0.135 & 0.096 & 0.055 & 0.272 & 0.193 & 0.056 & 0.992 \\
\hline & 1.707 & 1.753 & 1.789 & 1.856 & 1.898 & 1.953 & 2.018 & \\
\hline & 0.002 & 0.004 & 0.005 & 0.011 & 0.002 & 0.002 & 0.005 & \\
\hline \multirow[t]{5}{*}{3} & 12045.350 & 11372.600 & 10705.620 & 10045.380 & 9392.840 & 8748.965 & 8114.723 & \\
\hline & 0.830 & 0.879 & 0.934 & 0.996 & 1.065 & 1.143 & 1.232 & \\
\hline & 0.078 & 0.202 & 0.011 & 0.162 & 0.000 & 0.199 & 0.241 & 0.892 \\
\hline & 1.673 & 1.715 & 1.777 & 1.800 & 2.111 & 1.910 & 1.963 & \\
\hline & 0.003 & 0.001 & 0.021 & 0.001 & 0.097 & 0.004 & 0.001 & \\
\hline \multirow[t]{5}{*}{4} & 12604.260 & 11931.510 & 11264.540 & 10604.290 & 9951.750 & 9307.875 & 8673.634 & \\
\hline & 0.793 & 0.838 & 0.888 & 0.943 & 1.005 & 1.074 & 1.153 & \\
\hline & 0.028 & 0.143 & 0.119 & 0.012 & 0.140 & 0.024 & 0.115 & 0.582 \\
\hline & 1.641 & 1.680 & 1.724 & 1.742 & 1.810 & 1.832 & 1.924 & \\
\hline & 0.005 & 0.002 & 0.004 & 0.023 & 0.002 & 0.020 & 0.008 & \\
\hline \multirow[t]{5}{*}{5} & 13157.920 & 12485.170 & 11818.190 & 11157.950 & 10505.410 & 9861.532 & 9227.291 & \\
\hline & 0.760 & 0.801 & 0.846 & 0.896 & 0.952 & 1.014 & 1.084 & \\
\hline & 0.009 & 0.073 & 0.152 & 0.038 & 0.062 & 0.080 & 0.071 & 0.485 \\
\hline & 1.611 & 1.648 & 1.688 & 1.737 & 1.762 & 1.822 & 1.850 & \\
\hline & 0.006 & 0.004 & 0.000 & 0.011 & 0.008 & 0.007 & 0.009 & \\
\hline \multirow[t]{5}{*}{6} & 13706.360 & 13033.620 & 12366.640 & 11706.400 & 11053.850 & 10409.980 & 9775.738 & \\
\hline & 0.730 & 0.767 & 0.809 & 0.854 & 0.905 & 0.961 & 1.023 & \\
\hline & 0.003 & 0.030 & 0.109 & 0.116 & 0.002 & 0.100 & 0.028 & 0.387 \\
\hline & 1.583 & 1.618 & 1.656 & 1.697 & 1.788 & 1.773 & 1.838 & \\
\hline & 0.008 & 0.005 & 0.002 & 0.003 & 0.054 & 0.003 & 0.016 & \\
\hline$\Sigma_{v^{\prime}} \boldsymbol{q}_{v^{\prime} v^{\prime \prime}}$ & 0.999 & 0.983 & 0.900 & 0.715 & 0.605 & 0.622 & 0.514 & \\
\hline
\end{tabular}

For each $v^{\prime} \rightarrow v^{\prime \prime}$ The listed quantities are $v_{v^{\prime} v^{\prime \prime}}\left(\mathrm{cm}^{-1}\right), \lambda_{v^{\prime} v^{\prime \prime}}(\mu \mathrm{m}), q_{v^{\prime} v^{\prime \prime}}, \bar{r}_{v^{\prime} v^{\prime \prime}}(\AA),\left|1-Y_{v^{\prime} v^{\prime \prime}}^{(3)}\right|$

Table 3: Transition Probability Parameters for $K^{3} \Phi_{4}-X^{3} \Phi_{4}$ band system

\begin{tabular}{|c|c|c|c|c|c|}
\hline $\mathbf{v}^{\prime} \backslash \mathbf{v}^{\prime \prime}$ & $\mathbf{0}$ & 1 & 2 & 3 & $\Sigma_{\mathbf{v}^{\prime \prime}} \mathbf{q}_{\mathbf{v}^{\prime} \mathbf{v}^{\prime \prime}}$ \\
\hline \multirow{5}{*}{$\mathbf{0}$} & 18909.843 & 18237.096 & 17570.119 & & \\
\hline & 0.529 & 0.548 & 0.569 & & \\
\hline & 0.905 & 0.093 & 0.001 & & 1.000 \\
\hline & 1.753 & 1.887 & 2.183 & & \\
\hline & 0.002 & 0.012 & 0.064 & & \\
\hline \multirow{5}{*}{1} & 19575.003 & 18902.256 & 18235.279 & & \\
\hline & 0.511 & 0.529 & 0.548 & & \\
\hline & 0.085 & 0.740 & 0.170 & & 0.996 \\
\hline & 1.621 & 1.764 & 1.897 & & \\
\hline & 0.020 & 0.005 & 0.011 & & \\
\hline \multirow{5}{*}{2} & 20231.605 & 19558.858 & 18891.880 & 18231.637 & \\
\hline & 0.494 & 0.511 & 0.529 & 0.549 & \\
\hline & 0.008 & 0.143 & 0.608 & 0.234 & 0.993 \\
\hline & 1.549 & 1.624 & 1.777 & 1.907 & \\
\hline & 0.022 & 0.020 & 0.009 & 0.009 & \\
\hline \multirow[t]{5}{*}{3} & 20879.644 & 20206.897 & 19539.919 & 18879.677 & \\
\hline & 0.479 & 0.495 & 0.512 & 0.530 & \\
\hline & 0.001 & 0.021 & 0.180 & 0.502 & 0.703 \\
\hline & 1.491 & 1.549 & 1.628 & 1.790 & \\
\hline & 0.026 & 0.023 & 0.019 & 0.014 & \\
\hline$\Sigma_{\mathbf{v}^{\prime}} \mathbf{q}_{\mathbf{v}^{\prime} \mathbf{v}^{\prime \prime}}$ & 1.000 & 0.997 & 0.960 & 0.735 & \\
\hline
\end{tabular}


Table 4: Transition Probability Parameters for $L^{3} \Phi_{4}-X^{3} \Phi_{4}$ band system

\begin{tabular}{|c|c|c|c|c|c|}
\hline $\boldsymbol{v}^{\prime} \backslash \boldsymbol{v}^{\prime \prime}$ & $\mathbf{0}$ & 1 & 2 & 3 & $\Sigma_{\boldsymbol{v}^{\prime \prime}} \boldsymbol{q}_{\boldsymbol{v}^{\prime} \boldsymbol{v}^{\prime \prime}}$ \\
\hline \multirow[t]{5}{*}{$\mathbf{0}$} & 19236.420 & 18563.670 & & & \\
\hline & 0.520 & 0.539 & & & \\
\hline & 0.914 & 0.084 & & & 0.998 \\
\hline & 1.753 & 1.897 & & & \\
\hline & 0.002 & 0.014 & & & \\
\hline \multirow[t]{5}{*}{1} & 19877.270 & 19204.520 & 18537.540 & 17877.300 & \\
\hline & 0.503 & 0.521 & 0.539 & 0.559 & \\
\hline & 0.081 & 0.762 & 0.152 & 0.006 & 1.000 \\
\hline & 1.615 & 1.763 & 1.908 & 2.105 & \\
\hline & 0.022 & 0.006 & 0.013 & 0.034 & \\
\hline \multirow[t]{5}{*}{2} & 20512.600 & 19839.850 & 19172.870 & 18512.630 & \\
\hline & 0.488 & 0.504 & 0.522 & 0.540 & \\
\hline & 0.006 & 0.139 & 0.639 & 0.207 & 0.990 \\
\hline & 1.507 & 1.621 & 1.774 & 1.920 & \\
\hline & 0.042 & 0.021 & 0.010 & 0.012 & \\
\hline \multirow[t]{5}{*}{3} & & 20469.660 & 19802.680 & 19142.440 & \\
\hline & & 0.489 & 0.505 & 0.522 & \\
\hline & & 0.014 & 0.180 & 0.540 & 0.734 \\
\hline & & 1.511 & 1.626 & 1.785 & \\
\hline & & 0.042 & 0.020 & 0.014 & \\
\hline$\Sigma_{v^{\prime}} \boldsymbol{q}_{v^{\prime} v^{\prime \prime}}$ & 1.000 & 0.999 & 0.971 & 0.752 & \\
\hline
\end{tabular}

Table 5: Transition Probability Parameters For $[20.6]^{3} \Gamma_{5}-X^{3} \Phi_{4}$ band system

\begin{tabular}{|c|c|c|c|c|c|}
\hline $\mathbf{v}^{\prime} \backslash \mathbf{v}^{\prime \prime}$ & $\mathbf{0}$ & $\mathbf{1}$ & $\mathbf{2}$ & $\mathbf{3}$ & $\boldsymbol{\Sigma}_{\boldsymbol{v}^{\prime \prime}} \boldsymbol{q}_{\boldsymbol{v}^{\prime} \boldsymbol{v}^{\prime \prime}}$ \\
\hline $\mathbf{0}$ & 20655.560 & 19982.820 & 19315.840 & & \\
\hline & 0.484 & 0.500 & 0.518 & & \\
\hline & $\mathbf{0 . 7 6 1}$ & 0.217 & 0.022 & & 0.999 \\
\hline & 1.762 & 1.847 & 1.947 & & \\
\hline $\mathbf{1}$ & 0.002 & 0.004 & 0.011 & & \\
\hline & 21279.300 & 20606.560 & 19939.580 & & \\
\hline & 0.470 & 0.485 & 0.502 & & \\
\hline & 0.201 & $\mathbf{0 . 4 1 4}$ & $\mathbf{0 . 3 3 0}$ & 0.053 & 0.998 \\
\hline & 1.683 & 1.773 & 1.858 & 1.957 & \\
\hline $\mathbf{2}$ & 0.005 & 0.007 & 0.003 & 0.010 & \\
\hline & 21896.240 & 21223.500 & 20556.520 & 19896.280 & \\
\hline & 0.457 & 0.471 & 0.487 & 0.503 & \\
\hline & 0.034 & $\mathbf{0 . 2 7 9}$ & 0.214 & $\mathbf{0 . 3 8 1}$ & 0.908 \\
\hline & 1.616 & 1.689 & 1.786 & 1.869 & \\
\hline $\mathbf{3}$ & 0.012 & 0.004 & 0.013 & 0.001 & \\
\hline & 22506.380 & 21833.640 & 21166.660 & 20506.420 & \\
\hline & 0.444 & 0.458 & 0.472 & 0.488 & \\
\hline & 0.004 & 0.075 & $\mathbf{0 . 2 9 5}$ & 0.103 & 0.477 \\
\hline & 1.548 & 1.620 & 1.695 & 1.802 & \\
\hline & 0.020 & 0.010 & 0.002 & 0.023 & \\
\hline$\Sigma_{\boldsymbol{v}} \boldsymbol{q}_{\boldsymbol{v}^{\prime} \boldsymbol{v} \boldsymbol{v}^{\prime \prime}}$ & 0.999599 & 0.98523 & 0.8603 & 0.5366 & \\
\hline & & & & & \\
\hline
\end{tabular}


Table 6: Transition Probability parameters for $[34.7]^{3} \Gamma_{5}-X^{3} \Phi_{4}$ band system

\begin{tabular}{|c|c|c|c|c|c|}
\hline $\boldsymbol{v}^{\prime} \backslash \boldsymbol{v}^{\prime \prime}$ & $\mathbf{0}$ & $\mathbf{1}$ & $\mathbf{2}$ & $\mathbf{3}$ & $\boldsymbol{\Sigma}_{\boldsymbol{v}^{\prime \prime}} \boldsymbol{q}_{\boldsymbol{v}^{\prime} \boldsymbol{v}^{\prime \prime}}$ \\
\hline $\mathbf{0}$ & 34697.740 & 34024.990 & & & \\
\hline & 0.288 & 0.294 & & & 1.000 \\
\hline & $\mathbf{0 . 9 6 7}$ & 0.033 & & & \\
\hline & 1.748 & 1.976 & & & \\
\hline $\mathbf{1}$ & 0.002 & 0.035 & & & \\
\hline & 35376.170 & 34703.420 & 34036.440 & & 1.000 \\
\hline & 0.283 & 0.288 & 0.294 & & \\
\hline & 0.031 & $\mathbf{0 . 9 0 8}$ & $\mathbf{0 . 0 6 2}$ & & \\
\hline & 1.518 & 1.758 & 1.989 & & \\
\hline & 0.076 & 0.005 & 0.034 & & \\
\hline $\mathbf{2}$ & 36047.380 & 35374.630 & 34707.650 & 34047.410 & \\
\hline & 0.277 & 0.283 & 0.288 & 0.294 & \\
\hline & 0.002 & 0.054 & $\mathbf{0 . 8 5 8}$ & $\mathbf{0 . 0 8 6}$ & 1.000 \\
\hline & 1.472 & 1.513 & 1.769 & 2.003 & \\
\hline & 0.032 & 0.084 & 0.009 & 0.034 & \\
\hline $\mathbf{3}$ & 36711.370 & 36038.620 & 35371.640 & 34711.400 & \\
\hline & 0.272 & 0.278 & 0.283 & 0.288 & \\
\hline & 0.000 & 0.005 & 0.071 & $\mathbf{0 . 8 1 7}$ & 0.893 \\
\hline & 1.403 & 1.469 & 1.508 & 1.781 & \\
\hline & 0.049 & 0.033 & 0.093 & 0.012 & \\
\hline $\boldsymbol{\Sigma}_{\boldsymbol{v}}^{\prime} \boldsymbol{q}_{\boldsymbol{v}^{\prime} \boldsymbol{v}^{\prime \prime}}$ & 1.000 & 1.000 & 0.991 & 0.903 & \\
\hline & & & & & \\
\hline
\end{tabular}

Table 7: FCFs for $\mathrm{G}^{3} \Phi_{4}-\mathrm{X}^{3} \Phi_{4}$ band system

\begin{tabular}{|c|c|c|c|c|c|c|c|c|}
\hline $\boldsymbol{v}^{\prime} \backslash \boldsymbol{v}^{\prime \prime}$ & $\mathbf{0}$ & $\mathbf{1}$ & $\mathbf{2}$ & $\mathbf{3}$ & $\mathbf{4}$ & $\mathbf{5}$ & $\mathbf{6}$ & $\boldsymbol{\Sigma}_{\boldsymbol{v}^{\prime \prime}} \boldsymbol{q}_{\boldsymbol{v}^{\prime} \boldsymbol{v}^{\prime \prime}}$ \\
\hline $\mathbf{0}$ & 0.357 & $\mathbf{0 . 4 0 0}$ & 0.187 & 0.048 & 0.007 & 0.001 & 0.000 & \\
\hline & 0.357 & $\mathbf{0 . 4 0 0}$ & 0.187 & 0.048 & 0.007 & 0.001 & 0.000 & \\
\hline & 0.357 & $\mathbf{0 . 4 0 0}$ & 0.188 & 0.048 & 0.007 & 0.001 & 0.000 & 1.000 \\
\hline & 0.357 & $\mathbf{0 . 4 0 0}$ & 0.187 & 0.048 & 0.007 & 0.001 & 0.000 & \\
\hline & 0.346 & $\mathbf{0 . 3 8 2}$ & 0.195 & 0.061 & 0.013 & 0.002 & 0.000 & \\
\hline $\mathbf{1}$ & $\mathbf{0 . 3 3 9}$ & 0.000 & 0.225 & $\mathbf{0 . 2 8 5}$ & 0.122 & 0.026 & 0.003 & \\
\hline & $\mathbf{0 . 3 3 9}$ & 0.000 & 0.225 & $\mathbf{0 . 2 8 5}$ & 0.122 & 0.026 & 0.003 & \\
\hline & $\mathbf{0 . 3 3 9}$ & 0.000 & 0.225 & $\mathbf{0 . 2 8 5}$ & 0.122 & 0.026 & 0.003 & 1.000 \\
\hline & $\mathbf{0 . 3 3 9}$ & 0.000 & 0.225 & $\mathbf{0 . 2 8 5}$ & 0.122 & 0.026 & 0.003 & \\
\hline & $\mathbf{0 . 3 4 5}$ & 0.002 & 0.180 & $\mathbf{0 . 2 6 7}$ & 0.147 & 0.047 & 0.010 & \\
\hline $\mathbf{2}$ & $\mathbf{0 . 1 8 6}$ & 0.135 & 0.096 & 0.055 & $\mathbf{0 . 2 7 1}$ & 0.193 & 0.056 & \\
\hline & $\mathbf{0 . 1 8 6}$ & 0.135 & 0.096 & 0.055 & $\mathbf{0 . 2 7 1}$ & 0.193 & 0.056 & \\
\hline & $\mathbf{0 . 1 8 6}$ & 0.135 & 0.096 & 0.055 & $\mathbf{0 . 2 7 2}$ & 0.193 & 0.056 & 0.992 \\
\hline & $\mathbf{0 . 1 8 6}$ & 0.135 & 0.096 & 0.055 & $\mathbf{0 . 2 7 2}$ & 0.193 & 0.056 & \\
\hline & $\mathbf{0 . 1 9 2}$ & 0.130 & 0.115 & 0.021 & $\mathbf{0 . 2 0 5}$ & 0.207 & 0.096 & \\
\hline $\mathbf{3}$ & 0.078 & $\mathbf{0 . 2 0 2}$ & 0.011 & 0.162 & 0.000 & 0.198 & $\mathbf{0 . 2 4 1}$ & \\
\hline & 0.078 & $\mathbf{0 . 2 0 2}$ & 0.011 & 0.162 & 0.000 & 0.198 & $\mathbf{0 . 2 4 1}$ & \\
\hline & 0.078 & $\mathbf{0 . 2 0 2}$ & 0.011 & 0.162 & 0.000 & 0.199 & $\mathbf{0 . 2 4 1}$ & 0.892 \\
\hline & 0.078 & $\mathbf{0 . 2 0 2}$ & 0.011 & 0.162 & 0.000 & 0.199 & $\mathbf{0 . 2 4 1}$ & \\
\hline & 0.079 & $\mathbf{0 . 2 1 1}$ & 0.005 & 0.166 & 0.011 & 0.097 & $\mathbf{0 . 2 1 3}$ & \\
\hline $\mathbf{4}$ & 0.028 & $\mathbf{0 . 1 4 3}$ & 0.119 & 0.012 & 0.140 & 0.024 & 0.115 & \\
\hline & 0.028 & $\mathbf{0 . 1 4 3}$ & 0.119 & 0.012 & 0.140 & 0.024 & 0.115 & \\
\hline & 0.028 & $\mathbf{0 . 1 4 3}$ & 0.119 & 0.012 & 0.140 & 0.024 & 0.115 & 0.582 \\
\hline & 0.028 & $\mathbf{0 . 1 4 3}$ & 0.119 & 0.012 & 0.140 & 0.024 & 0.116 & \\
\hline & 0.027 & $\mathbf{0 . 1 5 2}$ & 0.116 & 0.027 & 0.110 & 0.074 & 0.019 & \\
\hline $\mathbf{5}$ & 0.009 & 0.073 & $\mathbf{0 . 1 5 2}$ & 0.038 & 0.062 & 0.080 & 0.071 & \\
\hline & 0.009 & 0.073 & $\mathbf{0 . 1 5 2}$ & 0.038 & 0.062 & 0.080 & 0.071 & \\
\hline & 0.009 & 0.073 & $\mathbf{0 . 1 5 2}$ & 0.038 & 0.062 & 0.080 & 0.071 & 0.485 \\
\hline & 0.009 & 0.073 & $\mathbf{0 . 1 5 2}$ & 0.038 & 0.061 & 0.080 & 0.071 & \\
\hline & & & & & & & & \\
\hline & & & & \\
\hline & & & \\
\hline & & &
\end{tabular}




\begin{tabular}{|c|c|c|c|c|c|c|c|c|}
\hline & 0.008 & 0.076 & $\mathbf{0 . 1 6 3}$ & 0.026 & 0.091 & 0.034 & 0.122 & \\
\hline $\mathbf{6}$ & 0.003 & 0.030 & 0.109 & $\mathbf{0 . 1 1 6}$ & 0.002 & 0.100 & 0.028 & \\
\hline & 0.003 & 0.030 & 0.109 & $\mathbf{0 . 1 1 6}$ & 0.002 & 0.100 & 0.028 & \\
\hline & 0.003 & 0.030 & 0.109 & $\mathbf{0 . 1 1 6}$ & 0.002 & 0.100 & 0.028 & 0.387 \\
\hline & 0.003 & 0.030 & 0.109 & $\mathbf{0 . 1 1 6}$ & 0.002 & 0.100 & 0.028 & \\
\hline & 0.002 & 0.031 & 0.120 & $\mathbf{0 . 1 1 5}$ & 0.000 & 0.117 & 0.000 & \\
\hline $\boldsymbol{\Sigma}_{\boldsymbol{v}^{\prime}} \boldsymbol{q}_{\boldsymbol{v}^{\prime} \boldsymbol{v}^{\prime \prime}}$ & 0.999 & 0.983 & 0.900 & 0.715 & 0.605 & 0.622 & 0.514 & \\
\hline
\end{tabular}

The result order is respectively for Le Roy, Tele, Espy, Jarmain and Felenbok

Table 8: $r$-Centroids for $\mathrm{G}^{3} \Phi_{4}-\mathrm{X}^{3} \Phi_{4}$ band system

\begin{tabular}{|c|c|c|c|c|c|c|c|}
\hline $\boldsymbol{v}^{\prime} \backslash \boldsymbol{v}^{\prime \prime}$ & $\mathbf{0}$ & $\mathbf{1}$ & $\mathbf{2}$ & $\mathbf{3}$ & $\mathbf{4}$ & $\mathbf{5}$ & $\mathbf{6}$ \\
\hline $\mathbf{0}$ & 1.783 & 1.829 & 1.879 & 1.935 & 1.998 & 2.072 & 2.174 \\
\hline & 1.783 & 1.829 & 1.879 & 1.935 & 1.998 & 2.072 & 2.174 \\
\hline & 1.783 & 1.829 & 1.879 & 1.935 & 1.998 & 2.072 & 2.174 \\
\hline & 1.783 & 1.829 & 1.879 & 1.935 & 1.998 & 2.072 & 2.174 \\
\hline & 1.781 & 1.826 & 1.873 & 1.920 & 1.969 & 2.017 & 2.066 \\
\hline $\mathbf{1}$ & 1.743 & 1.640 & 1.840 & 1.889 & 1.944 & 2.008 & 2.084 \\
\hline & 1.743 & 1.640 & 1.840 & 1.889 & 1.944 & 2.008 & 2.084 \\
\hline & 1.743 & 1.640 & 1.840 & 1.889 & 1.944 & 2.008 & 2.084 \\
\hline & 1.743 & 1.639 & 1.840 & 1.888 & 1.944 & 2.008 & 2.084 \\
\hline & 1.743 & 1.767 & 1.834 & 1.880 & 1.927 & 1.975 & 2.023 \\
\hline $\mathbf{2}$ & 1.707 & 1.753 & 1.789 & 1.856 & 1.898 & 1.954 & 2.018 \\
\hline & 1.707 & 1.753 & 1.789 & 1.856 & 1.898 & 1.953 & 2.018 \\
\hline & 1.707 & 1.753 & 1.789 & 1.856 & 1.898 & 1.953 & 2.018 \\
\hline & 1.707 & 1.753 & 1.789 & 1.856 & 1.898 & 1.953 & 2.018 \\
\hline & 1.705 & 1.751 & 1.792 & 1.845 & 1.887 & 1.933 & 1.981 \\
\hline $\mathbf{3}$ & 1.673 & 1.715 & 1.777 & 1.800 & 2.110 & 1.910 & 1.963 \\
\hline & 1.673 & 1.715 & 1.777 & 1.800 & 2.111 & 1.910 & 1.963 \\
\hline & 1.673 & 1.715 & 1.777 & 1.800 & 2.111 & 1.910 & 1.963 \\
\hline & 1.673 & 1.715 & 1.777 & 1.800 & 2.109 & 1.910 & 1.963 \\
\hline & 1.670 & 1.714 & 1.772 & 1.801 & 1.839 & 1.894 & 1.940 \\
\hline $\mathbf{4}$ & 1.641 & 1.680 & 1.724 & 1.742 & 1.810 & 1.832 & 1.924 \\
\hline & 1.641 & 1.680 & 1.724 & 1.742 & 1.810 & 1.832 & 1.924 \\
\hline & 1.641 & 1.680 & 1.724 & 1.742 & 1.810 & 1.832 & 1.924 \\
\hline & 1.641 & 1.680 & 1.724 & 1.742 & 1.810 & 1.832 & 1.924 \\
\hline & 1.636 & 1.678 & 1.723 & 1.757 & 1.809 & 1.851 & 1.904 \\
\hline $\mathbf{5}$ & 1.611 & 1.648 & 1.688 & 1.737 & 1.762 & 1.822 & 1.850 \\
\hline & 1.611 & 1.648 & 1.688 & 1.737 & 1.762 & 1.822 & 1.850 \\
\hline & 1.611 & 1.648 & 1.688 & 1.737 & 1.762 & 1.822 & 1.850 \\
\hline & 1.611 & 1.648 & 1.688 & 1.737 & 1.762 & 1.822 & 1.850 \\
\hline & 1.604 & 1.644 & 1.686 & 1.735 & 1.768 & 1.819 & 1.859 \\
\hline $\mathbf{6}$ & 1.583 & 1.618 & 1.656 & 1.697 & 1.788 & 1.773 & 1.838 \\
\hline & 1.583 & 1.618 & 1.656 & 1.697 & 1.788 & 1.773 & 1.838 \\
\hline & 1.583 & 1.618 & 1.656 & 1.697 & 1.788 & 1.773 & 1.838 \\
\hline & 1.583 & 1.618 & 1.655 & 1.697 & 1.787 & 1.773 & 1.838 \\
\hline & 1.574 & 1.612 & 1.652 & 1.695 & 1.669 & 1.777 & 1.892 \\
\hline & 075 & & & \\
\hline & & &
\end{tabular}

The result order is respectively for Le Roy, Tele, Espy, Jarmain and Felenbok 
Table 9: Wave numbers, Wavelengths and $r$-centroids of few bands of $\Delta v=3$ sequence of $\mathrm{G}^{3} \Phi_{4}-X^{3} \Phi_{4}$

\begin{tabular}{|c|c|c|c|}
\hline$\left(v^{\prime}, v^{\prime \prime}\right)$ & $\bar{r}_{v^{\prime} v^{\prime \prime}}(\AA)$ & $v_{v^{\prime} v^{\prime \prime}}\left(\mathrm{cm}^{-1}\right)$ & $\lambda_{v^{\prime} v^{\prime \prime}}(\AA)$ \\
\hline$(3,0)$ & 1.6727 & 12045.35 & 8302.0 \\
\hline$(4,1)$ & 1.6802 & 11931.51 & 8381.2 \\
\hline$(5,2)$ & 1.6882 & 11818.19 & 8461.5 \\
\hline$(6,3)$ & 1.6967 & 11706.40 & 8542.3 \\
\hline$(7,4)$ & 1.7065 & 11597.14 & 8622.8 \\
\hline$(8,5)$ & 1.7203 & 11491.42 & 8702.1 \\
\hline
\end{tabular}

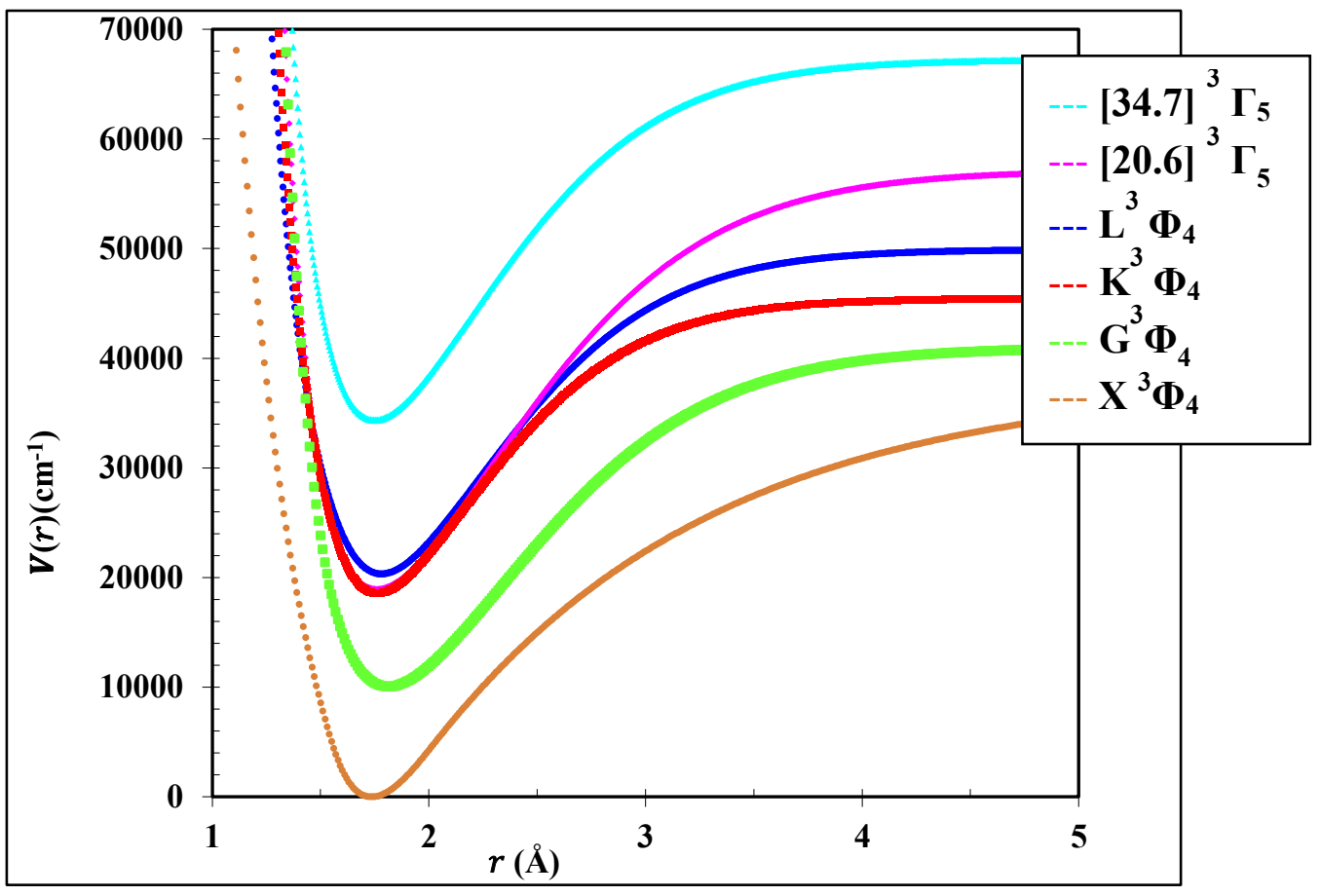

Fig. 1: PECs for the $\mathrm{X}^{3} \Phi_{4}, \mathrm{G}^{3} \Phi_{4}, \mathrm{~K}{ }^{3} \Phi_{4}, \mathrm{~L}^{3} \Phi_{4},[20.6]^{3} \Gamma_{5} \& \quad[34.7]^{3} \Gamma_{5}$ States of ${ }^{59} \mathrm{Co}^{19} \mathrm{~F}$ 


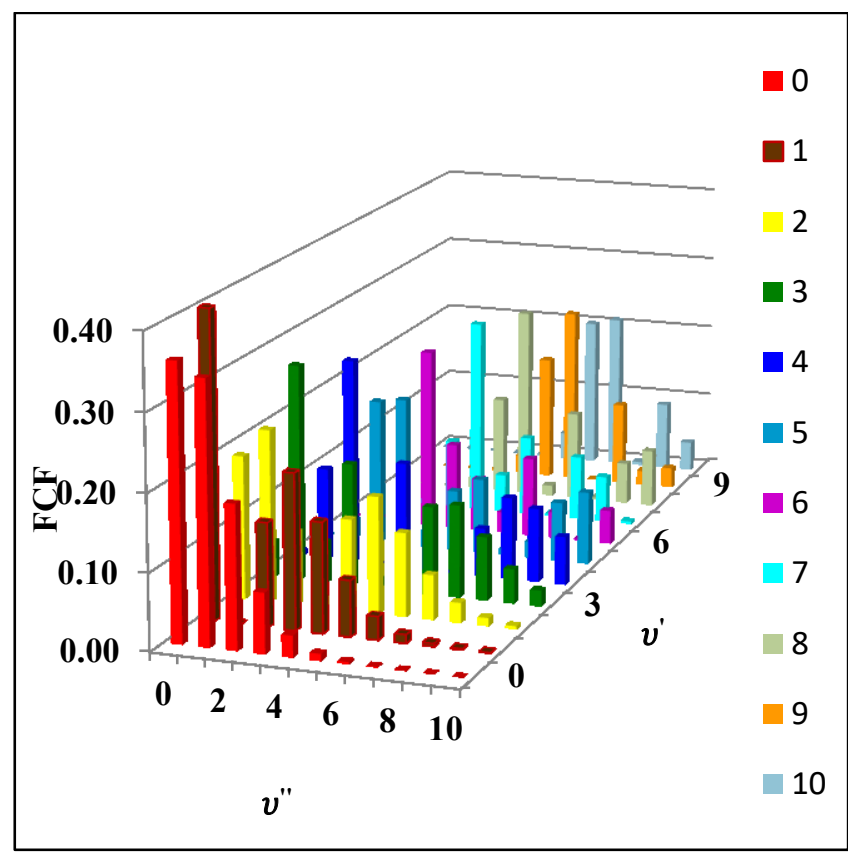

Fig. 2: 3-D Pars of FCF's for the G-X transition

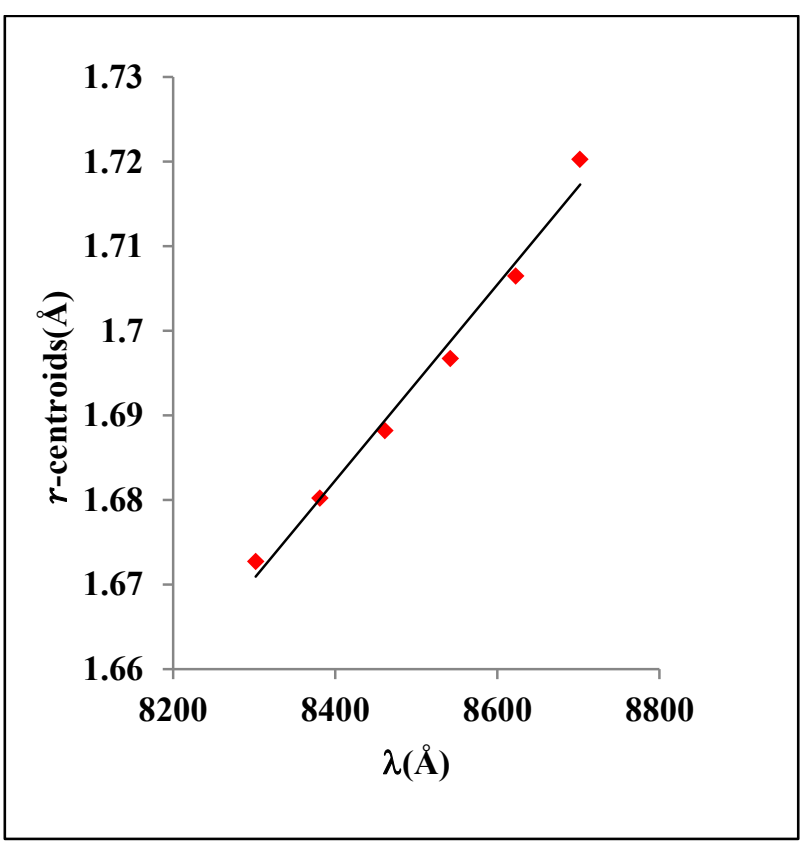

Fig. 3: Variation of $r$-centroids with $\lambda$ for $\Delta v=3$ sequence of G-X

\section{CONCLUSION}

FCFs and $r$-centroids for the G-X, K-X, L-X,[20.6 $]^{3} \Gamma_{5}-\mathrm{X}$ and $[34.7]^{3} \Gamma_{5}-\mathrm{X}$ systems of cobalt monofluorid have been calculated for the first time using the Morse and RKR potentials. The generated values using Morse potential show's deviation with those generated by RKR potentials even for low v-level transitions, however, both sets of the results follow the same pattern. This deviation does not depend on Morse program that we used but on nature of Morse model. We also checked the Morse result using the standard program LEVEL8 which gives fairly the same result presented here. The calculated values of FCFs may help in the search and experimental identification of new bands in these systems. On the other hand, the $r$-centroid values will be helpfull in studies of variations in electronic transition moment with internuclear distance as well as in the search for other molecular properties as a function of $r$.

\section{Acknowledgement}

We gratefully acknowledge Prof. R. Le Roy for making his programs available on his website[22]. We are also thankfull to Prof. P. J. Espy and Prof. A. Antić-Jovanović for their helpful interaction with us.

\section{References}

[1] Kuzmanović M. , Antić-Jovanović A., Bojović V., Dimitrije Pesić,“ Frank-Condon factors and $r$-centroids for the B-X bands of ${ }^{10} \mathrm{~B}^{18} \mathrm{O}$ and ${ }^{11} \mathrm{~B}^{18} \mathrm{O}$ molecules", J.Serb.Chem.Soc.70, 727 (2005); 10.2298/JSC0505727K

[2] Nicholls R.W."“ Franck-Condon factor formulae for astrophysical and other molecules”, Astrophys.J.Suppl.Ser. 47, 279(1981); 10.1086/190760

[3] Fraser P.A."“ a Method Of Determining The Electronic Transition Moment for Diatomic Molecules”, Can.J.Phys. 32, 515 (1954); 10.1139/p54-054

[4] Nicholls R.W., and Jarmain W.R., "vibrational transition probabilities to high quantum numbers for the nitrogen first and second positive band systems", Can.J.Phys. 32, 201 (1954); $10.1139 / \mathrm{p} 54-018$ 
[5] Nicholls R.W., and Jarmain W.R.," $r$-Centroids: Average Internuclear Separations Associated with Molecular Bands”, Proc.Phys.Soc.A. 69, 253 (1956); 10.1088/0370$1298 / 69 / 3 / 308$

[6] Nicholls R.W., Amani M., and Mandelman M.,“ The $r$-centroid concept revisited”, Can.J.Phys. 79, 611 (2001); 10.1139/p01-017

[7] Yersin H., "Electronic and Vibronic Spectra of Transition Metal Complexes II", SpringerVerlag Berlin Heidelberg (1997).

[8] Zhaoxia Zhang, Jingru Guo, Xufeng Yu, Junfeng Zheng, Yang Chen,“ The laser-induced fluorescence spectroscopy of $\mathrm{CoF}$ in the ultra violet region”, J.Mol.Spectrosc. 244, 117(2007); 10.1016/j.jms.2007.05.003

[9] Ram R.S., Bernath P.F., Davis S.P., "Fourier Transform Emission Spectroscopy of the $[10.3]^{3} \Phi_{i}-X^{3} \Phi_{i}$ System of CoF”, J.Mol.Spectrosc. 173, 158(1995); 10.1006/jmsp. 1995.1226 .

[10] Xiaopeng Zhang, Jingru Guo, Tingting Wang, Linsen Pei, Yang Chen, and Congxiang Chen,“ Visible laser spectroscopy of cobalt monofluoride", J.Mol.Spectrosc. 220, 209(2003); 10.1016/S0022-2852(03)00130-9

[11] Adam A.G., Hamilton W.D., "A Hund's Case (a) Analysis of the $[18.8]^{3} \Phi_{i}-X^{3} \Phi_{\text {i }}$ Electronic Transition of CoF”, J.Mol.Spectrosc. 206, 139. (2001); 10.1006/jmsp.2000.8292

[12] R.S. Ram, Bernath P.F, Davis S.P, "The low-lying electronic states of CoF", J.Chem.Phys. 104, 6949(1996); 10.1063/1.471411

[13] Herzberg G., "Spectra of Diatomic Molecules", Van Nostrand Reinhold Company, New York, (1950).

[14] Benesch W., Vanderslice J.T. and Tilford S.G., " $r$-centroid calculations for observed and permitted transitions in $\mathrm{N}_{2}$ ", J.Mol.Spectrosc. 36,464 (1970); 10.1016/0022-2852(70)90221-3

[15] Drake J., Nicholls R.W., "The $r$-centroid approximation and molecular spectra", Chem.Phys.Letts. 3, 457 (1969); 10.1016/0009-2614(69)80167-3

[16] Morse P.M., "Diatomic Molecules According to the Wave Mechanics. II. Vibrational Levels", Phys.Rev. 34, 57(1929); 10.1103/PhysRev.34.57

[17] Rydberg, R. "Graphische Darstellung einiger bandenspektroskopischer Ergebnisse", Z.Phys. 73, 376(1931); 10.1007/BF01341146.

[18] Klein O., "Zur Berechnung von Potentialkurven für zweiatomige Moleküle mit Hilfe von Spektraltermen", Z.Phys. 76, 226(1932); 10.1007/BF01341814

[19] Rees A.L.G., "The calculation of potential-energy curves from band-spectroscopic data", Proc.Phys.Soc. 59, 998 (1947); 10.1088/0959-5309/59/6/310

[20] Nadhem Q. M., Supriya Behere and S. H. Behere, "Realistic Franck-Condon Factors and rCentroids for the $a$-X Bands of CS Molecule”,IOSR-J.Appl.Phys. 7,3(2015); 10.9790/B07340312

[21] Le Roy R.J., RKR1 2.0: A Computer Program Implementing the First-Order RKR Method for Determining Diatomic Molecule Potential Energy Curves, University of Waterloo Chemical Physics Research Report CP-657R (2004).

[22] Le Roy R.J., LEVEL 8.0: A Computer Program for Solving the Radial Schrödinger Equation for Bound and Quasibound Levels, University of Waterloo Chemical Physics Research Report CP-663 (2007); see http://leroy.uwaterloo.ca/programs/.

[23] Telle H., Telle, U., "FCFRKR - A procedure to evaluate Franck-Condon type integrals for diatomic molecules", Comput.Phys.Commun. 28, 1 (1982); 10.1016/0010-4655(82)90059-5

[24] Gilmore. F. R., Laher. R.R., Espy. P.J,“ Franck-Condon Factors, r-Centroids, Electronic Transition Moments, and Einstein Coefficients for Many Nitrogen and Oxygen Band Systems", J.Phys.Chem.Ref.Data. 21, 1005 (1992); 10.1063/1.555910

[25] Jarmain W.R. and McCallum J.C., "TRAPRB, A Computer Programme for Molecular Transitions", Department of Physics, University of Western Ontario, (1970).

[26] Felenbok P., C. R. Acad. Sci. 256, 2, 334 (1963); https://goo.g1/2C9QuU

[27] Nicholls R.W., "Franck-Condon factors to high vibrational quantum numbers $\mathrm{I} . \mathrm{N}_{2}$ and $\mathrm{N}_{2}{ }^{+ \text {", }}$ J. Res. NBS. 65 A, 451(1961); 10.6028/jres.065A.047 
[28] Nicholls R.W., “ 'Condon Parabola' in Molecular Spectra”, Nature. 193, 966 (1962); 10.1038/193966a0

[29] Pekeris C.L., "The Rotation-Vibration Coupling in Diatomic Molecules", Phys.Rev. 45, 98 (1934); 10.1103/PhysRev.45.98

[30] McCallum J.C., "Computational study of the accuracy of the r-centroid approximation", J.Quant.Spectrosc.Radiat.Transfer. 21,563 (1979); 10.1016/0022-4073(79)90097-9 